\title{
The emerging role of FDG PET/CT in rectal cancer management: is it time to use the technique for early prognostication?
}

\author{
Luca Tagliabue
}

Published online: 23 February 2013

(C) Springer-Verlag Berlin Heidelberg 2013

Colorectal cancer is the second leading cause of death and the fourth most commonly diagnosed cancer in the USA [1]. One form, rectal cancer, defined as cancer growing within $12 \mathrm{~cm}$ of the anal verge [2], is challenging because of its unique anatomical characteristics, critical position and therapeutic options (aimed at preserving pelvic floor integrity, sphincter continence and genitourinary functions).

\section{General considerations and staging procedures}

When a patient is diagnosed with rectal cancer, staging procedures, performed to evaluate global and specific therapeutic risk, include: complete bowel endoscopy (to identify possible synchronous tumours), rigid proctoscopy (to establish the exact level of the lesion), carcinoembryonic antigen (CEA) test and a full clinical examination (to determine performance status). Moreover, for a complete evaluation of the disease, including possible distant metastases, a contrast-enhanced (ce) CT of the thorax, abdomen and pelvis, with administration of oral and IV contrast media, must also be performed. In some circumstances, rectal wall invasion and the presence of pathologic lymph nodes can be assessed by endoscopic ultrasound (EUS) and ceMRI of the pelvis [3]. In a meta-analysis of 90 studies, performed to compare the clinical performance of EUS, MRI and CT in rectal cancer staging, Bipat et al. found EUS and MRI to show similar sensitivity in the diagnosis of muscularis propria invasion (94\%), while EUS was more accurate in the evaluation of local tumour invasion ( 86 vs $69 \%$ ); ceCT, on the other hand, is not considered a gold standard

L. Tagliabue $(\bowtie)$

Nuclear Medicine Unit, Department of Diagnostic Imaging,

"San Paolo" Hospital, via A. di Rudinì 8, 20142 Milan, Italy

e-mail: luca.tagliabue@ao-sanpaolo.it investigation for evaluating tumour wall penetration. Local lymph node involvement, which constitutes a major challenge in rectal cancer staging, can be effectively evaluated by using all three methods, even though only ceCT and MRI provide information on distant node status and metastasis [4]. An advantage of MRI is that it allows better evaluation of the mesorectum, while a relative disadvantage of EUS is its relatively high dependence on operator skill.

\section{Pathologic considerations}

Histopathologic analysis of resected lesions is also an important part of staging, both initial staging and restaging after neoadjuvant therapy. The final report should contain the following information: a gross description of the tumour burden, the grade of the cancer, the depth of penetration and invasion of adjacent structures $(\mathrm{T})$, the number of lymph nodes examined (not fewer than 10 and ideally between 12 and 18), the number of metastatic lymph nodes present, if any $(\mathrm{N})$, the presence of any metastasis to other organs or to non-regional lymph nodes, the status of margins, the effect of neoadjuvant treatment and the presence, if any, of lymphovascular invasion, perineural invasion and extranodal cancer deposits $[5,6]$. Unlike other colonic segments, the rectum is not or is only partially covered by the peritoneum: therefore, particular attention should be paid to evaluation of circumferential margins of resection (CMR). Both in naive patients and in those undergoing neoadjuvant chemoradiotherapy (CRT), thorough assessment of CMR status greatly influences post-surgical decisions because it is a strong predictor of clinical outcome, including local recurrence and overall survival (OS) [7-9]. For this reason, the 7th edition of the American Joint Committee on Cancer (AJCC) Cancer Staging Manual, in accordance with the most recent College of American Pathologists Guidelines, requires that information about pathologic 
response to neoadjuvant CRT be included in the report, indicated with " $y$ ". Many scales for grading response have been suggested: Rödel et al., for example, proposed a scale of 0 (no pathologic response) to 4 (complete pathologic response) and suggested that patients with a complete response [tumour regression grading (TRG) 4] or an intermediate pathologic response (TRG 2+3) should be considered "responders", likely to have improved disease-free survival (DFS) after preoperative CRT [10].

\section{Therapeutic options}

As mentioned above, the treatment of rectal cancer is particularly challenging. Because local recurrence is known to be associated with a poorer prognosis [11], and also because of the need to preserve locoregional functions, the achievement of optimal local control through surgical removal of the tumour, when this is possible, is considered the most important goal in rectal cancer treatment. Unfortunately, whereas surgery for local control of disease is more feasible in colonic segments other than the rectum, allowing clinical strategies to focus on metastasis prevention, in the rectum, complete surgical eradication may be impossible, particularly in the case of larger tumours.

Organ-sparing procedures, preserving sphincter and genitourinary functions, should always be preferred but, unfortunately, are not always possible. In locally advanced rectal tumours (stage II: T3-T4, node-negative with deep muscle wall invasion and stage III: node-positive without other metastases), preoperative CRT may result in tumour downstaging, decreasing tumour bulk, improving tumour exposure and making sphincter-preserving surgery possible, especially when initial staging indicates the need for a more aggressive approach.

\section{Neoadjuvant and adjuvant CRT in resectable rectal cancer}

As indicated, local control of rectal disease can be difficult to achieve due to the presence of functionally important structures close to, or involved in, the tumour. Compared with analysis of pathology specimens, preoperative staging procedures have limitations and pitfalls, mainly related to suboptimal diagnostic performances of EUS and ceMRI (which can miss microscopic extranodal and intramural tumour deposits) but in some cases related to operator ability [12]. This suggests that many patients who might benefit from a neoadjuvant approach could be understaged. Nowadays, most major guidelines recommend a neoadjuvant CRT regimen in all cT3 N0 patients; combined treatment modalities, including fluorouracil-based regimens, radiotherapy, surgery and adjuvant regimens, are considered appropriate for the vast majority of stage II/III patients. There is currently some debate over whether radiotherapy should be administered before or after surgery. In the German Rectal Cancer Study, a large, prospective randomized study (CAO/ARO/AIO-94 Trial), preoperative CRT was clearly shown to reduce local recurrences (6 vs $14 \%$, $p=0.006$ ) without affecting progression-free survival (PFS) and 10-year OS [13]. Adding chemotherapy to radiotherapy regimens offers remarkable advantages. Preliminary results of a phase III trial suggest that adding a chemotherapy approach to radiotherapy alone can result in better local control due to significant reductions in tumour size, pTN stage and lymphatic, perivascular and neural invasion [14]. More consolidated results in this field are provided by the European Organization for Research and Treatment of Cancer Radiation Oncology Group (EORTC 22921): their results indicate that only locally advanced rectal cancer patients (stage II and III) administered neoadjuvant CRT that resulted in downstaging of $\mathrm{T}$ to $\mathrm{T} 0-\mathrm{T} 2$ (ypT0-2) will benefit, in terms of DFS and OS, from post-surgical continuation of the same neoadjuvant chemotherapeutic regimen (fluorouracil-based, with or without leucovorin), compared with patients who showed minimal or no modification of their initial staging (ypT3-ypT4) [15]. Another interesting observation, based on retrospective, non-randomized and uncontrolled studies, was made by other authors (HabrGama et al. [16] and Glynne-Jones et al. [17]), who postulated a wait-and-see strategy in patients showing a complete pathologic response (ypT0 in up to $20 \%$ in some series) after neoadjuvant CRT, an approach that could reduce surgery-related mortality and morbidity. For the moment, these latter findings are to be considered purely anecdotal; the level of evidence is weak and further investigations are needed just before they can be "translated" into clinical recommendations for everyday practice.

As we have seen, different staging systems exist for locally advanced rectal cancer, and a wide range of options for treating it, associated with variable responses. In this setting, another important question emerges:

\section{Is there a role for molecular imaging, and in particular for ${ }^{18}$ F-fluorodeoxyglucose (FDG) positron emission tomography (PET)/CT, in the management of rectal cancer?}

According to authoritative guidelines [18], PET/CT scanning is not routinely indicated either in the staging or in the follow-up of rectal cancer. Conversely, PET/CT is indicated in the presence of serial CEA elevation and resectable metastases, in order to identify other localizations and avoid, as far as possible, "blinded, CEA-directed, laparotomies" 
[19]. A recent meta-analysis by Brush et al. produced insufficient evidence to support the routine use of FDG PET/CT in primary colorectal cancer, and only a small amount of evidence supporting its use in the preoperative staging of recurrent and metastatic colorectal cancer. Moreover, the same authors performed an economic evaluation and found that FDG PET/CT, used as an add-on imaging device, is cost-effective in the preoperative staging of recurrent colon cancer, recurrent rectal cancer and metastatic disease, but not in primary staging. They concluded that further randomized controlled trials with a concurrent economic evaluation are probably needed [20].

In 2007 Hindié et al. published an editorial in the European Journal of Nuclear Medicine and Molecular Imaging [21] addressing the question of the role of $\mathrm{PET} / \mathrm{CT}$ in the management of rectal (and oesophageal) cancer. They posed some interesting questions: What place does CRT currently have in the treatment of gastrointestinal tract tumours? Why might response evaluation by FDG PET be important in this setting? What is the optimal way to perform FDG PET, given the limitations of FDG? Has the time come for "standardization" of PET/CT methods with a view to starting trials involving PET-based management? They concluded that response prediction can be essential for planning and providing optimal therapy and that PET will have to be incorporated into future trials. Identification of the optimal PET criteria for response prediction is needed, and these criteria will need to be harmonized.

In recent years a growing body of evidence has accumulated in the literature, attesting to the increasing importance of PET/CT in predicting response to therapy and advocating the use of metabolic imaging in early evaluation of response to therapy (so-called "interim" evaluation), not only in rectal tumours. In patients with rectal cancer who receive neoadjuvant CRT, rates of complete pathologic response range from 5 to $44 \%$, while rates of pathologic complete response of nodal disease in patients with pathologic complete response of the primary tumour range from 0 to $15 \%$. Given the possibility of performing "interim" metabolic and functional evaluation, experts are now beginning to debate the question of what to do in patients with a pathologic complete response also demonstrated by FDG PET/CT; it is still necessary to expose them to the rigours of aggressive surgery or would a wait-and-see strategy be more appropriate [22]? In an interesting paper, just published in the European Journal of Nuclear Medicine and Molecular Imaging, Murcia Duréndez et al. stated that PET/CT is a reliable technique for assessing response to neoadjuvant radiochemotherapy (RCT) in locally advanced rectal cancer, with a view to considering more conservative surgical treatment [23]. It seems clear that interim evaluation by PET/CT could influence primary local treatment in rectal cancer. But does it also have a role in early prognosis? In this issue of the European Journal of Nuclear Medicine and Molecular Imaging, [24] et al. confirm a role for ${ }^{18} \mathrm{~F}-\mathrm{FDG}$ PET/CT imaging in predicting histopathologic response and clinical long-term outcomes in locally advanced rectal cancer. Moreover, they demonstrate that a standardized uptake value (SUV)-based evaluation of tumour metabolism at 5 weeks after the end of radiotherapy (performed as a part of a neoadjuvant regimen) is able not only to predict the regression of tumours in pathology specimens, but also to prospectively identify a strong correlation with patients' longterm outcome, between responders and non-responders, in terms of PFS and OS. In particular, they underline the prognostic utility, in these patients, of SUV reduction, with an SUV cut-off of $65 \%$.

\section{Is the time ripe for SUV-based diagnostic algorithms?}

A review of the literature on the possible role of SUVs in clinical practice is outside the scope of this editorial. Generally speaking, SUV (maximum SUV, mean SUV, total glycolytic activity and so on) is a semi-quantitative measure of tissue glucose utilization in cancer. For this reason, SUV and SUV modifications have been extensively used in different types of cancer for diagnosis, staging, prognosis and evaluation of local or distant relapse [24, 25], although the superiority of SUV versus visual analysis has not always been demonstrated [26]. Even though higher-grade and less differentiated tumours are generally associated with higher levels of FDG accumulation, in diagnostic settings there can be a significant overlap in SUV values between cancer and other non-malignant diseases [27]; moreover, the existence of tumours characterized by low degrees of FDG uptake is well known [28]. For these reasons, SUV analysis may have only a partial role in the diagnostic setting. Conversely, when a diagnosis of cancer has been established, metabolic evaluations, even if performed using a relatively nonspecific tracer such as FDG, seem to give the best results. In this setting, the possibility of having a precise measure of tumour viability may have important repercussions for the therapy [29]. Clearly, if a change of therapy is possible, it could be extremely useful to have a cut-off value of a metabolic index, such as SUV, that could help in decisionmaking. From this perspective, the work of Calvo et al. is worthy of attention and calls for further, SUV-based and clinically oriented investigations. We are well aware that the various problems surrounding SUVs-for example, problems in SUV calculation and reproducibility, clearly set out in the European Journal of Nuclear Medicine and Molecular Imaging editorial [22] - are still far from resolved. A partial solution to these problems, as outlined by Calvo et al. in their work, could possibly lie in correct standardization of the method and the accumulation of 
experience, by single centres, in order to increase confidence and familiarity with imaging and its clinical implications.

\section{Conclusion}

Methods for the evaluation of tumour burden response to therapies, giving us the first and unique in vivo images of tumour viability, are opening a new era in cancer treatment. We know-and metabolic imaging provides daily confirmation of this - that every cancer is different, every patient is different and the final, sometimes dramatic, conclusion of our patients' clinical courses is the result of close interactions, complex and still only partially understood, between cancer biology, host behaviour and response to therapies. We trust that investigation of metabolic changes of tumours will help us to improve our knowledge of cancer biology and treatment, with the ultimate aim of curing the individual patient of his particular cancer and giving him more life, while reducing side effects and toxicities and making the best possible use of economic resources.

"Consider the seed of your generation:

You are not born to live like animals

But to pursue virtue and possess knowledge"

D. Alighieri. Divine Comedy. Hell XXVI 118-120.

\section{References}

1. Siegel R, Naishadham D, Jemal A. Cancer statistics, 2012. CA Cancer J Clin 2012;62(1):10-29. doi:10.3322/caac.20138.

2. Kapiteijn E, Marijnen CA, Nagtegaal ID, Putter H, Steup WH, Wiggers T, et al. Preoperative radiotherapy combined with total mesorectal excision for resectable rectal cancer. N Engl J Med 2001;345(9):638-46.

3. Bartram C, Brown G. Endorectal ultrasound and magnetic resonance imaging in rectal cancer staging. Gastroenterol Clin North Am 2002;31(3):827-39.

4. Bipat S, Glas AS, Slors FJ, Zwinderman AH, Bossuyt PM, Stoker J. Rectal cancer: local staging and assessment of lymph node involvement with endoluminal US, CT, and MR imaging-a meta-analysis. Radiology 2004;232(3):773-83.

5. Washington MK, Berlin J, Branton P, Burgart LJ, Carter DK, Fitzgibbons PL, et al. Protocol for the examination of specimens from patients with primary carcinoma of the colon and rectum. Arch Pathol Lab Med 2009;133(10):1539-51. doi:10.1043/1543-2165-133.10.1539.

6. Ueno H, Mochizuki H, Hashiguchi Y, Ishiguro M, Miyoshi M, Kajiwara Y, et al. Extramural cancer deposits without nodal structure in colorectal cancer: optimal categorization for prognostic staging. Am J Clin Pathol 2007;127(2):287-94.

7. Glynne-Jones R, Mawdsley S, Novell JR. The clinical significance of the circumferential resection margin following preoperative pelvic chemo-radiotherapy in rectal cancer: why we need a common language. Colorectal Dis 2006;8(9):800-7.
8. Adam IJ, Mohamdee MO, Martin IG, Scott N, Finan PJ, Johnston $\mathrm{D}$, et al. Role of circumferential margin involvement in the local recurrence of rectal cancer. Lancet 1994;344(8924):707-11.

9. Compton CC, Fielding LP, Burgart LJ, Conley B, Cooper HS, Hamilton SR, et al. Prognostic factors in colorectal cancer. College of American Pathologists Consensus Statement 1999. Arch Pathol Lab Med 2000;124(7):979-94.

10. Rödel C, Martus P, Papadoupolos T, Füzesi L, Klimpfinger M, Fietkau R, et al. Prognostic significance of tumor regression after preoperative chemoradiotherapy for rectal cancer. J Clin Oncol 2005;23:8688-96.

11. Wiig JN, Larsen SG, Giercksky KE. Operative treatment of locally recurrent rectal cancer. Recent Results Cancer Res 2005;165:13647.

12. Guillem JG, Díaz-González JA, Minsky BD, Valentini V, Jeong SY, Rodriguez-Bigas MA, et al. cT3N0 rectal cancer: potential overtreatment with preoperative chemoradiotherapy is warranted. J Clin Oncol 2008;26(3):368-73. doi:10.1200/JCO.2007.13.5434.

13. Sauer R, Becker H, Hohenberger W, Rödel C, Wittekind C, Fietkau $\mathrm{R}$, et al. Preoperative versus postoperative chemoradiotherapy for rectal cancer. N Engl J Med 2004;351(17):1731-40.

14. Bosset JF, Calais G, Mineur L, Maingon P, Radosevic-Jelic L, Daban A, et al. Enhanced tumorocidal effect of chemotherapy with preoperative radiotherapy for rectal cancer: preliminary resultsEORTC 22921. J Clin Oncol 2005;23(24):5620-7.

15. Collette L, Bosset JF, den Dulk M, Nguyen F, Mineur L, Maingon P, et al. Patients with curative resection of cT3-4 rectal cancer after preoperative radiotherapy or radiochemotherapy: does anybody benefit from adjuvant fluorouracilbased chemotherapy? A trial of the European Organisation for Research and Treatment of Cancer Radiation Oncology Group. J Clin Oncol 2007;25(28):4379-86.

16. Habr-Gama A, Perez RO, Nadalin W, Sabbaga J, Ribeiro Jr U, Silva e Sousa Jr AH, et al. Operative versus nonoperative treatment for stage 0 distal rectal cancer following chemoradiation therapy: long-term results. Ann Surg 2004;240(4):711-7. discussion 717-8.

17. Glynne-Jones R, Wallace M, Livingstone JI, Meyrick-Thomas J. Complete clinical response after preoperative chemoradiation in rectal cancer: is a "wait and see" policy justified? Dis Colon Rectum 2008;51(1):10-9. discussion 19-20.

18. NCCN Guideline Version 4.2013 www.NCCN.org.

19. Martin Jr EW, Minton JP, Carey LC. CEA-directed second-look surgery in the asymptomatic patient after primary resection of colorectal carcinoma. Ann Surg 1985;202(3):310-7.

20. Brush J, Boyd K, Chappell F, Crawford F, Dozier M, Fenwick E, et al. The value of FDG positron emission tomography/computerised tomography $(\mathrm{PET} / \mathrm{CT})$ in pre-operative staging of colorectal cancer: a systematic review and economic evaluation. Health Technol Assess 2011;15(35):1-192. doi:10.3310/hta15350. iii-iv.

21. Hindié E, Hennequin C, Moretti JL. Predicting response to chemoradiotherapy in rectal and oesophageal cancer with $18 \mathrm{~F}$ FDG: prognostic value and possible role in patient management. Eur J Nucl Med Mol Imaging 2007;34:1576-82. doi:10.1007/ s00259-007-0483-5.

22. Dedemadi G, Wexner SD. Complete response after neoadjuvant therapy in rectal cancer: to operate or not to operate? Dig Dis 2012;30 Suppl 2:109-17. doi:10.1159/000342039.

23. Murcia Duréndez MJ, Frutos Esteban L, Luján J, Frutos MD, Valero G, Navarro Fernández JL, et al. The value of (18)F-FDG $\mathrm{PET} / \mathrm{CT}$ for assessing the response to neoadjuvant therapy in locally advanced rectal cancer. Eur J Nucl Med Mol Imaging 2013;40(1):91-7. doi:10.1007/s00259-012-2257-y.

24. Calvo FA, ClV S, de la Mata D, Cabezón L, Gómez-Espí M, Alvarez E, et al. 18F-FDG PET/CT-based treatment response evaluation in locally advanced rectal cancer: a prospective 
validation of long-term outcomes. Eur J Nucl Med Mol Imaging. doi:10.1007/s00259-013-2341-y.

25. Hillner BE, Tosteson AN, Song Y, Tosteson TD, Onega T, Goodman DC, et al. Growth in the use of PET for six cancer types after coverage by medicare: additive or replacement? J Am Coll Radiol 2012;9(1):33-41. doi:10.1016/j.jacr.2011.06.019.

26. Klayton T, Li T, Yu JQ, Keller L, Cheng J, Cohen SJ, et al. The role of qualitative and quantitative analysis of F18-FDG positron emission tomography in predicting pathologic response following chemoradiotherapy in patients with esophageal carcinoma. J Gastrointest Cancer 2012;43(4):612-8. doi:10.1007/s12029-0129412-3.
27. Shreve PD, Anzai Y, Wahl RL. Pitfalls in oncologic diagnosis with FDG PET imaging: physiologic and benign variants. Radiographics 1999;19:61-77.

28. Ambrosini V, Nicolini S, Caroli P, Nanni C, Massaro A, Marzola $\mathrm{MC}$, et al. PET/CT imaging in different types of lung cancer: an overview. Eur J Radiol 2012;81(5):988-1001. doi:10.1016/ j.ejrad.2011.03.020.

29. Gallamini A, Patti C, Viviani S, Rossi A, Fiore F, Di Raimondo F, et al. Early chemotherapy intensification with BEACOPP in advancedstage Hodgkin lymphoma patients with a interim-PET positive after two ABVD courses. Br J Haematol 2011;152(5):551-60. doi:10.1111/j.1365-2141.2010.08485.x. 